\title{
Alcohol drinking, cigarette smoking, and the development of squamous cell carcinoma of the esophagus
}

\author{
Yoshihiko Maehara
}

Received: 9 February 2010/Published online: 12 March 2010

(C) Japan Society of Clinical Oncology 2010

Esophageal cancer is a highly aggressive cancer and the surgical treatment is extremely invasive. In Japan, the patient prognosis has improved remarkably due to advances in tumor diagnosis, operative techniques, perioperative management, and chemoradiotherapy; however, approximately half of the patients cannot be cured even after an esophagectomy $[1,2]$. Early detection, as well as prevention, is therefore important to avoid esophageal cancer deaths.

Both cigarette smoking and alcohol drinking are wellestablished risk factors for esophageal squamous cell carcinoma (ESCC). A dose relationship and a synergistic effect of these two factors have been reported regarding the risk for ESCC [3-5]. Also, heavy exposure to these factors is closely related to multiple occurrences of SCC in the upper aerodigestive tract (UADT), including the esophagus and head and neck regions [6]. Furthermore, individual cancer susceptibility differs due to polymorphisms of metabolic enzymes $[7,8]$. Regarding SCC in the UADT, a polymorphism of acetaldehyde dehydrogenase 2 (ALDH2) has been reported to be important not only for the development of cancer but also for multicentric carcinogenesis [9].

Various kinds of genetic abnormalities have been investigated in ESCC, including the activation of oncogenes and inactivation of tumor-suppressor genes, and a large body of knowledge exists concerning esophageal carcinogenesis [10]. However, there is little direct evidence showing a causal relationship between alcohol consumption and cigarette smoking and the genetic abnormalities observed in ESCC. Furthermore, the molecular mechanism

\section{Y. Maehara $(\bowtie)$}

Department of Surgery and Science,

Graduate School of Medical Sciences, Kyushu University,

3-1-1 Maidashi, Higashi-ku, Fukuoka 812-8582, Japan

e-mail: maehara@surg2.med.kyushu-u.ac.jp of the joint effect of tobacco and alcohol has not been reviewed in detail.

Against this background, Dr. Morita and colleagues review the clinical significance of tobacco and alcohol as risk factors for ESCC, and Dr. Toh and colleagues discuss the molecular mechanism of tobacco- and alcohol-inducing carcinogenesis of the esophagus.

\section{References}

1. Morita M, Yoshida R, Ikeda K et al (2008) Advances in esophageal cancer surgery in Japan: an analysis of 1000 consecutive patients treated at a single institute. Surgery 143:499-508

2. Toh Y, Sakaguchi Y, Ikeda O et al (2009) The triangulating stapling technique for cervical esophago anastomosis after esophagectomy. Surg Today 39:201-206

3. International Agency for Research on Cancer (1986) Tobacco smoking. In: IARC monographs on the evaluation of carcinogenic risks to humans, vol 38, IARC Lyon

4. International Agency for Research on Cancer (1988) Alcohol drinking. In: IARC monographs on the evaluation of carcinogenic risks to humans, vol 44. IARC, Lyon

5. Morita M, Saeki H, Mori M et al (2002) Risk factors for esophageal cancer and the multiple occurrence of carcinoma in the upper aerodigestive tract. Surgery 131:S1-S6

6. Morita M, Araki K, Saeki H et al (2003) Risk factors for multicentric occurrence of carcinoma in the upper aerodigestive tract-analysis with a serial histologic evaluation of the whole resected-esophagus including carcinoma. J Surg Oncol 83:216221

7. Yoshino I, Maehara Y (2007) Impact of smoking status on the biological behavior of lung cancer. Surg Today 37:725-734

8. Seitz HK, Stickel F (2007) Molecular mechanisms of alcohol mediated carcinogenesis. Nat Rev Cancer 7:599-612

9. Muto M, Takahashi M, Ohtsu A et al (2005) Risk of multiple squamous cell carcinoma in the esophagus and the head and neck region. Carcinogenesis 26:1008-1012

10. Kuwano H, Kato H, Miyazaki T et al (2005) Genetic alterations in esophageal cancer. Surg Today 35:7-18 